\title{
Aging impairs vasodilatory responses in rats
}

\author{
Soon Yul Kim ${ }^{1}$, Jong Taek Park ${ }^{1}$, Jae Kyun Park ${ }^{1}$, Jeong Soo Lee ${ }^{2}$, and Jae Chan Choi ${ }^{1}$ \\ Department of Anesthesiology and Pain Medicine, ${ }^{1}$ Wonju College of Medicine, Yonsei University, Wonju, ${ }^{2}$ College of Medicine, \\ Yonsei University, Seoul, Korea
}

Background: Aging causes profound changes of stiffness and compliance in the cardiovascular system, which contributes to decreased cardiovascular reserve. Mechanisms of the underlying endothelial vasodilator dysfunction in vasodilator signaling pathways may occur at multiple sites within any of these pathways.

Methods: Age-related changes in the vasculature were investigated in adult young (3-6 months, Y) and old (2629 month, O) Wistar rats $(\mathrm{n}=6)$. The aortas were carefully dissected from the rat and cut into rings $1.5-2.0 \mathrm{~mm}$ in length to measure in vitro isometric tension. Vasorelaxant responses of aortic rings to acetylcholine (ACh), sodium nitroprusside (SNP) and P1075 were examined using Dose Response software (AD Instruments, Mountain View, CA). Results: Endothelium-dependent vasodilator function was impaired. The endothelium of aging rats impaired endothelial NO dependent vasodilation, but the machinery for vasodilation was not impaired.

Conclusions: Age-related NO-mediated vasorelaxation in the aging endothelium was inhibited and appears to be major mechanism of vascular change and impaired vascular regulation. (Korean J Anesthesiol 2011; 61: 506-510)

Key Words: Acetylcholine, Aging, Endothelium, Nitric Oxide, P1075, Sodium nitroprusside.

\section{Introduction}

Aging has been identified as the dominant risk factor for cardiovascular disease [1]. Further, animal studies have consistently reported, that age-related endothelial dysfunction in the vascular system plays an important role in cardiovascular disease [2-6]. Nitric oxide (NO) is an important factor involved in endothelial regulation of vascular function. In experimental animal models of aging, it has been reported that NO bioavailability is decreased, due to an increased production of superoxide anion and peroxynitrite $\left(\mathrm{ONOO}^{-}\right)[7,8]$. Vascular endothelial dysfunction occurs during the human aging process, and it is considered as a crucial event in the development of many vasculopathies. We investigated the underlying mechanisms of this process. The characteristic changes in aging vasculature include endothelial dysfunction. The mechanisms of underlying endothelial dysfunction, defined as impaired vasodilator response to agonists involve multiple signaling pathways. Age-associated vasodilator dysfunction results from an increased production of ROS which limits the bioavailability of NO or leads to a relative deficiency of substrate for the synthesis of NO. To test this hypothesis we measured vasoreactivity to endothelium dependent and independent vasodilators. We also examined the effect of vasodilator

Received: March 14, 2011. Revised: 1st, May 11, 2011; 2nd, June 22, 2011. Accepted: June 27, 2011.

Corresponding author: Soon Yul Kim, M.D., Department of Pain Medicine, Wonju College of Medicine, Yonsei University, 162, Ilsan-dong, Wonju 220-701, Korea. Tel: 82-33-741-1521, Fax: 82-33-742-8198, E-mail: soonyul@yonsei.ac.kr

(c) This is an open-access article distributed under the terms of the Creative Commons Attribution Non-Commercial License (http:// creativecommons.org/licenses/by-nc/3.0/), which permits unrestricted non-commercial use, distribution, and reproduction in any medium, provided the original work is properly cited. 
function in aging rats and young rats.

\section{Materials and Methods}

Wistar rats, 3-6 months of young age and 26-29 months of old age weighing $400-500 \mathrm{~g}$, were housed separately at $22^{\circ} \mathrm{C}$. The animals were fed regular rat chow and water ad libitum. The study was approved by the Institutional Animal Care and Use Committee. Animals were anesthetized with ketaminexylazine, and the aorta were rapidly removed and placed in ice-cold oxygenated Krebs-Ringer physiological buffer. The aortic rings with endothelium (ED+) were carefully dissected from the rat and cut into rings 1.5-2.0 $\mathrm{mm}$ in length for in vitro isometric tension measurements. A subset of aortic rings had the endothelium removed (ED-) by gentle rubbing of the luminal surface with a stainless steel rod. The completion of ED - was confirmed by the obliteration of the vasorelaxant response to the endothelium dependent agonist ACh. For the in vitro isometric tension measurements, aortic rings were suspended in $5 \mathrm{ml}$ organ chambers. The rings were mounted horizontally between two stainless steel stirrups. One of the stirrups was anchored to a micromanipulator and the other to a force transducer (FT-03) in a myograph (150 $\mu \mathrm{m}$ stirrups) (Mangus, Iwashiya Kishimoto Medical Instruments, Kyoto, Japan). All dose-response curves were performed on vessel rings beginning at their optimum resting tone. All of the aortic rings were stretched to $3,000 \mathrm{mg}$ in $500 \mathrm{mg}$ increments over a 1 hour period to optimize the contractile responses. Aortic rings were equilibrated in oxygenated cold modified Krebs-Ringer bicarbonate buffer with the following composition (mM); 118.3 $\mathrm{NaCl}, 4.7 \mathrm{KCl}, 1.2 \mathrm{MgSO}_{4}, 1.2 \mathrm{KH}_{2} \mathrm{PO}_{4}, 2.5 \mathrm{CaCl}_{2}, 25 \mathrm{NaHCO}_{3}$, 0.016 Ca-EDTA, and 11.1 glucose(control solution). Buffer was purged with $95 \% \mathrm{O}_{2}, 5 \% \mathrm{CO}_{2}$ and maintained at $\mathrm{pH} 7.4$ and $37^{\circ} \mathrm{C}$. Optimal resting tone was the minimum level of stretch allowed for the largest contractile response to $\mathrm{KCl}(60 \mathrm{mM})$. After aortic rings had been stretched to their optimal resting tone, contractile response to $60 \mathrm{mM} \mathrm{KCl}$ was determined. After removal of $\mathrm{KCl}$, all vessel rings were constricted by phenylephrine (PE: $10^{-9}$ to $10^{-4} \mathrm{M}$ ). To test the vasorelaxant capability of the vessel rings, they were preconstricted by $\mathrm{PE}\left(10^{-6} \mathrm{M}\right)$, and then vasorelaxant responses to acetylcholine (ACh:10 ${ }^{-9}$ to $10^{-5}$ $\mathrm{M})$, sodium nitroprusside (SNP:10 $0^{-9}$ to $10^{-5} \mathrm{M}$ ) and P1075 (10 $10^{-9}$ to $10^{-4} \mathrm{M}$ ) were determined in vessels from old and young rats. Data were collected on-line by using a MacLab system and analyzed by using Dose-Response software (AD Instruments, Mountain View, CA). In all experiments, vasoconstrictor responses were expressed as tension in milligrams. $\mathrm{ED}_{50}$ and maximal response $\left(\mathrm{E}_{\max }\right)$ were calculated by using nonlinear logistic regression analysis with the PRIZM ${ }^{\circledR}$ software (Graphpad, Mountain View, CA). All data are reported as means \pm SD. Statistical differences were determined by using a Student's t-test (paired t-test for within group comparisons and an unpaired t-test for between group comparisons).

\section{Results}

\section{Responses to PE}

PE $\left(10^{-9}\right.$ to $\left.10^{-4} \mathrm{M}\right)$ induced concentration-dependent contractions in isolated old and young rat aortic rings (Fig. 1). The concentration effect curves to PE statistically showed no difference of contraction in ED+ aortic rings from old and young rats $\left(\mathrm{E}_{\mathrm{max}}, 1,784 \pm 66 \mathrm{mg}\right.$ vs. $1,857 \pm 34 \mathrm{mg} ; \log \mathrm{ED}_{50},-7.22 \pm 0.12$ vs. $-7.27 \pm 0.06$ in old vs. young rats, respectively; $n=6$, Fig. 1 ). The concentration effect curves to $\mathrm{PE}$ also showed no difference of contraction in $\mathrm{ED}-$ aortic rings from old and young rats $\left(\mathrm{E}_{\max }\right.$, $2,222 \pm 47$ mg vs. $2,205 \pm 36 \mathrm{mg}$; $\log \mathrm{ED}_{50},-7.38 \pm 0.07$ vs. -7.61 \pm 0.06 in old vs. young rats respectively; $n=6$, Fig. 1 ).

\section{ACh, SNP, and P1075 evoked concentration-dependent relaxations}

ACh $\left(10^{-9}\right.$ to $\left.10^{-5} \mathrm{M}\right)$ evoked more relaxation of endothelium containing rings in young rats than old rats $\left(\mathrm{E}_{\max }, 72.4 \pm 2.8 \%\right.$ vs. $43.6 \pm 1.7 \%$; $\log \mathrm{ED}_{50},-7.700 \pm 0.17$ vs. $-7.491 \pm 0.12$ in young vs. old rats, respectively; $\mathrm{P}<0.05 ; \mathrm{n}=6$; Fig. 2). ACh-induced relaxation in aortic rings of old and young rats was abolished by denuding of the endothelium; Fig. 2). The relaxations induced by SNP $\left(10^{-9}\right.$ to $\left.10^{-5} \mathrm{M}\right)$ in the ED+ vascular rings showed some differences in both groups. ( $\mathrm{E}_{\max }, 101.9 \pm 2.4 \%$ vs. $84.2 \pm 1.9 \%$; $\log \mathrm{ED}_{50},-7.76 \pm 0.07$ vs. $-7.327 \pm 0.06$ in young vs. old rats, respectively; $\mathrm{n}=6$; Fig. 3$)$. But the relaxations to $\mathrm{P} 1075\left(10^{-9}\right.$ to $10^{-4}$

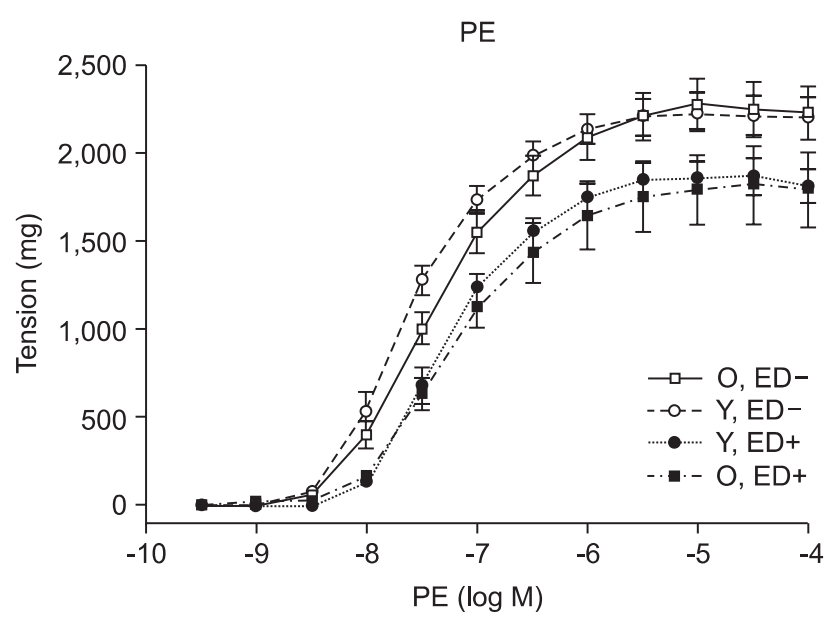

Fig. 1. PE dose-response curves of vessels from young and old rats (n $=6$ ). Responses are expressed as tension $(\mathrm{mg})$ and are presented as means $\pm \mathrm{SD}$ in $\mathrm{ED}+$ and $\mathrm{ED}-$ vessels. 


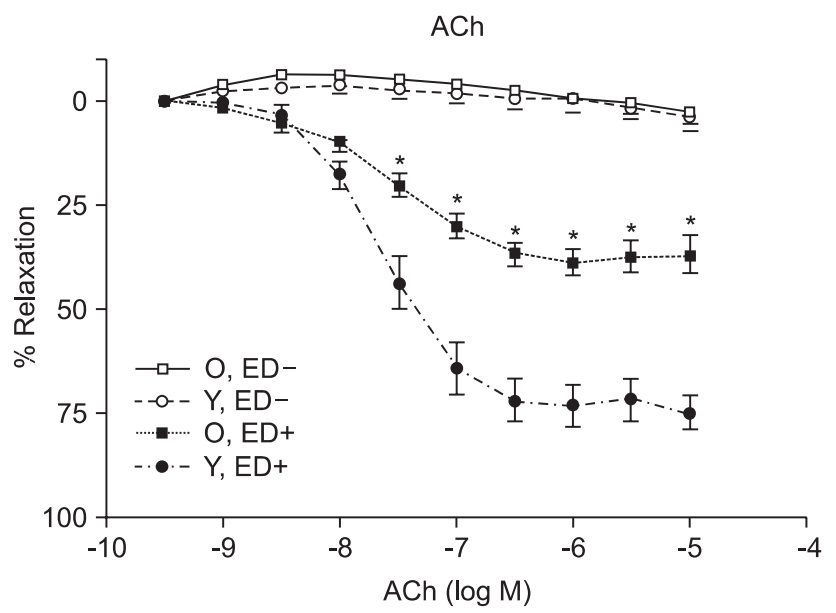

Fig. 2. ACh dose-response curves of vessels from young and old rats $(n=6)$. Vasorelaxant responses are expressed as a percentage of $E_{\max }$ after preconstriction with $\mathrm{PE}\left(10^{-6} \mathrm{M}\right)$ and are presented as means $\pm \mathrm{SD}$ in $\mathrm{ED}+$ and $\mathrm{ED}-$ vessels. The vasorelaxant $\mathrm{E}_{\max }$ to $\mathrm{ACh}$ was attenuated in $\mathrm{ED}+$ aortic rings from old rats $\left({ }^{*} \mathrm{P}<0.05\right)$, compared with young rats. There were no responses to $\mathrm{ACh}$ in $\mathrm{ED}-$ vessels.

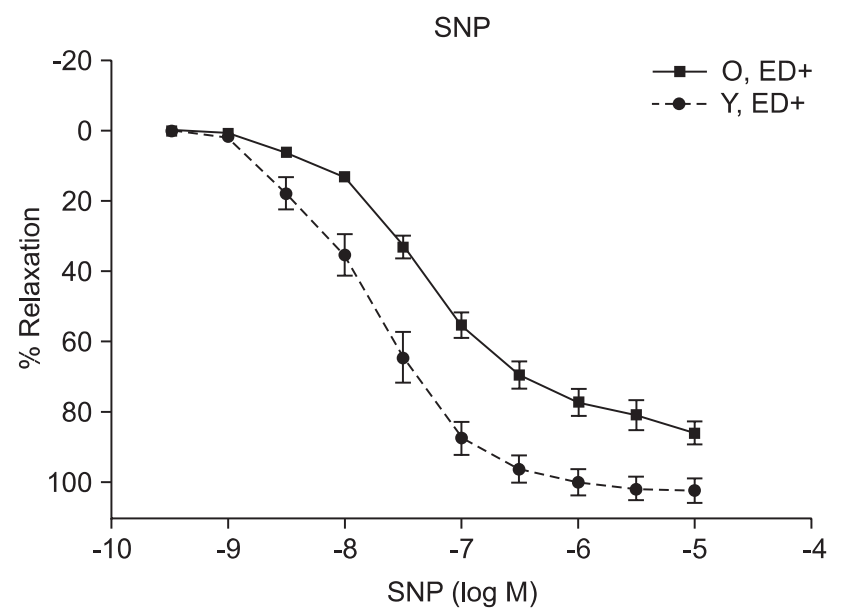

Fig. 3. SNP dose-response curves of vessels from young and old rats $(n=6)$. Vasorelaxant responses are expressed as a percentage of $E_{\max }$ after preconstriction with $\mathrm{PE}\left(10^{-6}\right)$ and are presented as means $\pm \mathrm{SD}$ in $\mathrm{ED}+$ vessels. The vasorelaxant $\mathrm{E}_{\max }$ to SNP was not significantly different in $\mathrm{ED}+$ aortic rings from old rats, compared with young rats.

$\mathrm{M})$ in $\mathrm{ED}+$ vascular rings were similar in both groups. $\left(\mathrm{E}_{\max }, 93.1\right.$ $\pm 5.0 \%$ vs. $103.0 \pm 5.5 \%$; $\log \mathrm{ED}_{50},-5.58 \pm 0.13$ vs. $-5.36 \pm 0.12$ in young vs. old rats, respectively, $\mathrm{n}=6$; Fig. 4). The relaxation by SNP of ED - vascular rings were similar in both groups $\left(\mathrm{E}_{\max }\right.$, $100.6 \pm 2.1 \%$ vs $99.0 \pm 1.9 \%$; $\log \mathrm{ED}_{50},-7.79 \pm 0.07$ vs. $-7.49 \pm 0.06$ in young vs. old rats, respectively; $n=6$; Fig. 5). The relaxation by $\mathrm{P} 1075$ of $\mathrm{ED}-$ vascular rings were also similar in both groups $\left(\mathrm{E}_{\max }, 90.7 \pm 7.3 \%\right.$ vs $98.2 \pm 9.7 \%$; $\log \mathrm{ED}_{50},-5.09 \pm 0.13$ vs. -4.04 \pm 0.14 ; in young vs. old rats, respectively; $n=6$; Fig. 4 ).

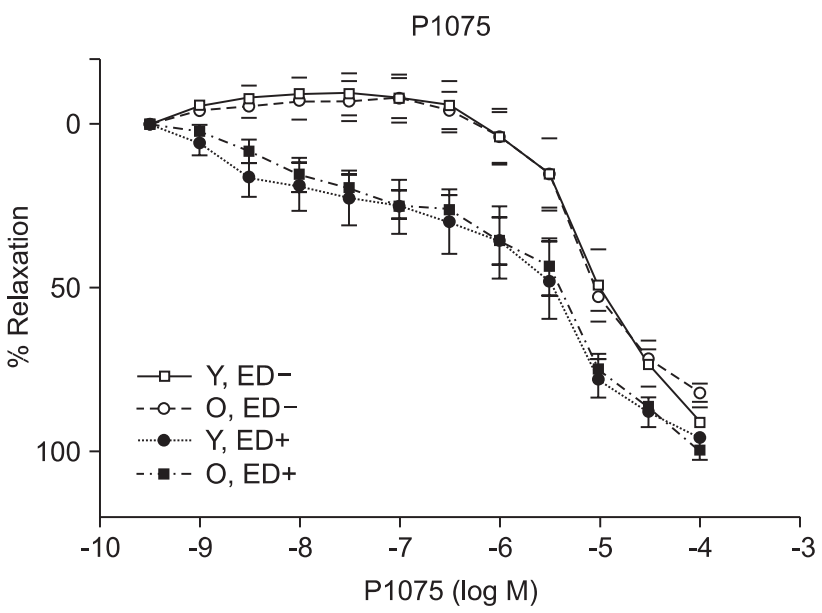

Fig. 4. P1075 dose-response curves of vessels from young and old rats $(n=6)$. Vasorelaxant responses are expressed as a percentage of $\mathrm{E}_{\max }$ after preconstriction with $\mathrm{PE}\left(10^{-6} \mathrm{M}\right)$ and are presented as means $\pm S D$ in $\mathrm{ED}+$ and $\mathrm{ED}-$ vessels. The vasorelaxant $\mathrm{E}_{\max }$ to P1075 was similar in ED + and ED - aortic from old and young rats.

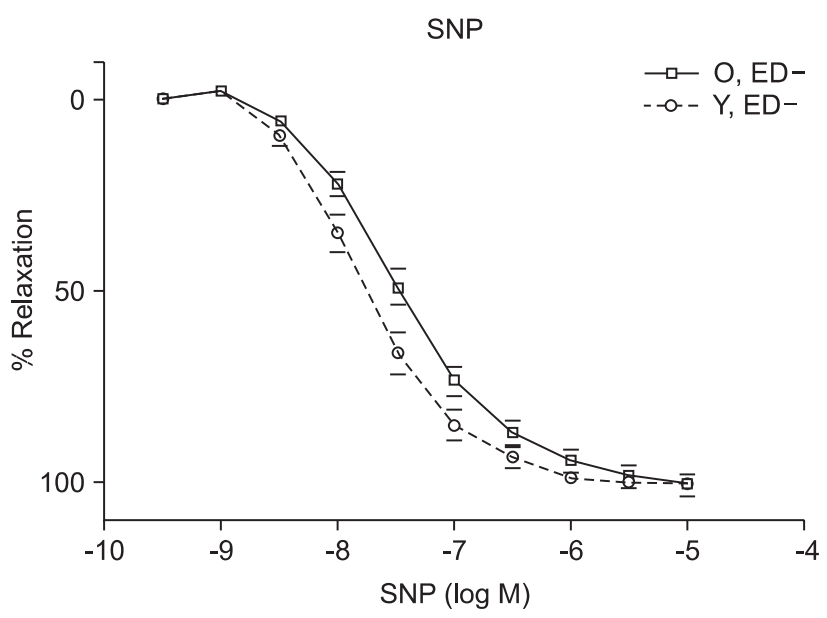

Fig. 5. SNP dose-response curves of vessels from young and old rats $(n=6)$. Vasorelaxant responses are expressed as a percentage of $E_{\max }$ after precontriction with $\mathrm{PE}\left(10^{-6}\right)$ and are presented as means $\pm \mathrm{SD}$ in ED- vessels. The vasorelaxant Emax to SNP was similar in EDaortic rings from old rats, compared with young rats.

\section{Discussion}

Numerous changes have been described in the cardiovascular system by aging. Examples of these changes include elastance and compliance changes in the arterial tree resulting in increased cardiac load as well as, impaired beta adrenergic receptor signaling in the heart and vasculature. Mechanisms underlying endothelial vasodilator dysfunction in the vasodilator signaling pathways may occur at multiple sites within any of these 
pathways. With regard to the nitric oxide (NO) pathway, abnormalities may occur secondarily to disturbances in the availability of substrate, receptor/G-protein signaling in the endothelial cell, the activity or expression of nitric oxide synthase (NOS), the bioavailability of NO and vascular smooth muscle signaling and relaxation. Decreases in the bioavailability of NO have been proposed as a mechanism of vasodilator/endothelial dysfunction in several diseases such as atherosclerosis, hypertension, and hypercholesterolemia. Moreover, endogenous inhibitors of NOS have recently been proposed as a possible mechanism for underlying vasodilator dysfunction in peripheral vascular disease and hypertension. Hypertension and hypercholesterolemia, two established risk factors for cardiovascular disease are common in the aging population. It is well recognized that endothelial dysfunction occurs in these two diseases. It has also becoming increasingly evident that vasodilator dysfunction occurs in aging independent of hypercholesterolemia and hypertension. Endothelial nitric oxide synthase (eNOS) is the enzyme expressed in endothelial cells that produces nitric oxide (NO), the most important vasodilatory molecule synthesized by the endothelium [9]. The most prominent mediators of endothelium dependent dilatation are NO and prostacyclin $\left(\mathrm{PGI}_{2}\right)$, which cause relaxation by stimulating guanylyl cyclase and adenylyl cyclase, respectively. Responses that are resistant to blockade of NOS and cyclooxygenase are associated with endothelium dependent hyperpolarization of vascular smooth muscle, and they are thought to be mediated by a distinct endothelium derived hyperpolarizing factor (EDHF)[10]. ACh produces dilatation in essentilly all vascular beds, including the pulmonary and coronary vascular beds [11]. Coronary vasodilatation may be elicited by either a cardiovascular reflex or by direct electrical stimulation of the vagus. The dilatation of vascular beds by choline esters is due to the presence of muscarinic receptors, primarily of the $\mathrm{M}_{3}$ subtype [12], despite the lack of apparent cholinergic innervation of most blood vessels. The muscarinic receptors which are responsible for relaxation are located on the endothelial cells of the vasculature; when these receptors are stimulated, the endothelial cells release NO (endothelium-derived relaxing factor)[13], which diffuses to adjacent smooth muscle cells and causes them to relax. If the endothelium is damaged, ACh can stimulate receptors on vascular smooth muscle cells and cause vasoconstriction. Although SNP has been known since 1850 and its hypotensive effect in human beings was described in 1929, its safety and usefulness for the short-term control of severe hypertension was not demonstrated until the mid-1950s. It is a nitro-vasodilator, metabolized by smooth muscle cells to its active metabolite, nitric oxide. NO activates guanylate cyclase, leading to the formation of cyclic GMP and vasodilatation [14]. The metabolic activation of SNP is catalyzed by a different NO generating system than nitroglycerin (NTG), which probably accounts for the difference in potency between these drugs at different vascular sites in addition to the fact that tolerance develops to NTG but not to SNP [15]. SNP dilates both arterioles and venules, and the hemodynamic response to its administration results from a combination of venous pooling and reduced arterial impedance. Its onset of action is within 30 seconds; the peak hypotensive effect occurs within 2 minutes, and when the infusion of the drug is stopped, the effect disappears within 3 minutes. The metabolism of SNP by smooth muscle is initiated by its reduction, which is followed by the release of cyanide and then nitric oxide [16]. The ATPsensitive K channel (KATP) opener, P1075\{N-cyano- $N^{\prime \prime}$-(1,1dimethylpropyl)- $N^{\prime \prime \prime}$-3-pyridylguanidine $\}$, is a cyanoguanidine drug that opens ATP-sensitive potassium channels producing peripheral vasodilatation of arterioles and a pinacidil analogue, an established opener of KATP channels $[17,18]$. Age related changes in the vasculature were investigated in old (26-29 months) and adult young (3-6 months) Wistar rats. Vasorelaxant responses were examined in aortic rings. PE-induced constriction of vessels harvested from old and young rats were not statistical difference (ED+; maximal response, 1,784 \pm 66 mg vs. $1,857 \pm 34 \mathrm{mg}$; $\log \mathrm{ED}_{50},-7.22 \pm 0.12$ vs. $-7.27 \pm 0.06$ in old vs. young rats, respectively; ED-; maximal response, 2,222 $\pm 47 \mathrm{mg}$ vs. $2,205 \pm 36 \mathrm{mg}$; $\log \mathrm{ED}_{50},-7.38 \pm 0.07$ vs. $-7.61 \pm$ 0.06 in old vs. young rats, respectively) (Fig. 1). Following PE preconstriction, ACh-induced does-dependent relaxation was profoundly attenuated in rings of old rats $\left(\mathrm{E}_{\max }, 72.4 \pm 2.8 \% \mathrm{vs}\right.$. $43.6 \pm 1.7 \%, \log \mathrm{ED}_{50},-7.700 \pm 0.17$ vs. $-7.491 \pm 0.12$ in old and young rats, respectively, $\mathrm{P}<0.05, \mathrm{n}=6$; Fig. 2). SNP, like endogenous NO, also activates cGMP-dependent mechanisms within vascular smooth muscle. SNP-induced relaxation was also impaired in ring from old rats $\left(\mathrm{ED}+; \mathrm{E}_{\max }, 101.9 \pm 2.4 \%\right.$ vs. $84.2 \pm 1.9 \%$; $\log \mathrm{ED}_{50},-7.76 \pm 0.07$ vs. $-7.327 \pm 0.06$ in young and old rats, respectively, $\mathrm{P}>0.05 ; \mathrm{n}=6$; Fig. 3 ). The impaired vasorelaxant response in $\mathrm{ED}+$ vessels from old rats was not observed in ED - vessels. The magnitude of the differential response in old versus young rats was similar in ED- rings. However, the dose response to SNP in ED + vessels from old rats was shifted to the right, suggesting a modulating (inhibitory) influence of the endothelium in the response to SNP. Relaxation of vascular smooth muscle is dependent on the integrity of the contractile/relaxation machinery. This system can be activated by a number of pathways including those dependent on NO/ cGMP, and pathways independent of NO but dependent on membrane polarization. To determine the specificity of our observation, we investigated changes in ATP-sensitive potassium $\left(\mathrm{K}^{+}{ }_{\text {ATP }}\right)$ channel activation, which were assessed by constructing dose responses with the channel opener P1075. 
The vasorelaxant responses to P1075 were identical in vessels from old and young rats (Fig. 4). Aortic rings have been utilized most frequently to assess vascular changes because of their accessibility and size. In ED+ aortic rings, both the ACh and SNP-curves were shifted to the right in all rats implying impaired vasorelaxant responses in old versus young. The maximal vasodilator response to ACh was profoundly impaired in aortic rings. In sum, removal of the endothelium abolished ACh response in old and young rats, and ACh vasorelaxant responses markedly attenuated in aortic rings from aging rats. SNP-induced vasorelaxation was no different in $\mathrm{ED}$ - rings from old and young rats. In contrast, vasorelaxant response to SNP is more attenuated in vessels from old rats in $\mathrm{ED}+$ rings. This suggests that the endothelium is inhibiting NO mediated vasorelaxation, possibly by decreasing its bioavalability. In order to determine whether machinery involved in the vasodilatory mechanisms which are impaired in vascular rings from aging rats, the vasorelaxant responses to P1075, a $\mathrm{K}^{+}$channel activator was tested . There was no significant difference in the vasorelaxant responses to hyperpolarization suggesting that vasorelaxant machinery distal to the NO pathway is intact. In conclusion, one mechanism of underlying age-related vascular changes was impaired in vascular regulation. In particular, aging endothelium was inhibiting NO-mediated vasorelaxation.

\section{References}

1. Lakatta EG, Levy D. Arterial and cardiac aging: major shareholders in cardiovascular disease enterprise: Part I: aging arteries: a 'set up' for vascular disease. Circulation 2003; 107: 139-46.

2. Hongo K, Nakagomi T, Kassell NF, Sasaki T, Lehman M, Vollmer DG, et al. Effects of aging and hypertension on endothelium-dependent vascular relaxation in rat carotid artery. Stroke 1988; 19: 892-7.

3. Koga T, Takata Y, Kobayashi K, Takishita S, Yamashita Y, Fujishima $\mathrm{M}$. Age and hypertension promote endothelium-dependent contractions to acetylcholine in the aorta of the rat. Hypertension 1989; 14: 542-8.

4. Küng CF, Lüscher TF. Different mechanisms of endothelial dysfunction with aging and hypertension in rat aorta. Hypertension 1995;
25: $194-200$

5. Matz RL, de Sotomayor MA, Schott C, Stoclet JC, Andriantsitohaina R. Vascular bed heterogeneity in age-related endothelial dysfunction with respect to NO and eicosanoids. Br J Pharmacol 2000; 131: 303 11.

6. Brandes RP, Fleming I, Busse R. Endothelial aging. Cardiovasc Res 2005; 66: 286-94.

7. Van der Loo B, Labugger R, Skepper JN, Bachschmid M, Kilo J, Powell JM, et al. Enhanced peroxynitrite formation is associated with vascular aging. J Exp Med 2000; 192: 1731-44.

8. Matz RL, Andriantsitohaina R. Age-related endothelial dysfunction: potential implications for pharmacotherapy. Drugs Aging 2003; 20 : 527-50.

9. Fleming I, Busse R. NO: the primary EDRF. J Mol Cell Cardiol 1999; 31: 5-14.

10. Nagao T, Vanhoutte PM. Hyperpolarization as a mechanism for endothelium dependent relaxations in porcine coronary artery. J Physiol 1992; 445: 355-67.

11. Hardman JG, Limbird LE, Molinoff PB, Ruddon RW, Gilman AG. Goodman \& Gilman's the pharmacological basis of therapeutics. 9th ed. Edited by the McGraw-Hill Companies: New York, Wonsiewicz and McCurdy publishers. 1996, p 143.

12. Boulanger CM, Morrison KJ, Vanhoutte PM. Mediation by M3muscarinic receptors of both endothelium-dependent contraction and relaxation to acetylcholine in the aorta of the spontaneously hypertensive rat. Br J Pharmacol 1994; 112: 519-24.

13. Moncada S, Palmer RM, Higgs EA. Nitric oxide: physiology, pathophysiology and pharmacology. Pharmacol Rev 1991; 43: 109-42.

14. Murad F. Cyclic guanosine monophosphate as a mediator of vasodilation. J Clin Invest 1986; 78: 1-5.

15. Kowaluk EA, Seth P, Fung HL. Metabolic activation of sodium nitroprusside to nitric oxide in vascular smooth muscle. J Pharmacol Exp Ther 1992; 262: 916-22.

16. Bates JN, Baker MT, Guerra R Jr, Harrison DG. Nitric oxide generation from nitroprusside by vascular tissue. Evidence that reduction of the nitroprusside anion and cyanide loss are required. Biochem Pharmacol 1991; 42 Suppl: S157-65.

17. Steinberg MI, Ertel P, Smallwood JK, Wyss V, Zimmerman K. The relation between vascular relaxant and cardiac electrophysiological effects of pinacidil. J Cardiovasc Pharmacol 1988; 12 Suppl: S30-40.

18. Quast U. Do the $\mathrm{K}+$ channel openers relax smooth muscle by opening K+ channels? Trends Pharmacol Sci 1993; 14: 332-7. 